\title{
Complex type seed variety identification and recognition using optimized image processing techniques
}

\author{
A.V. Seetha Lakshmi ${ }^{1}$ and H.Salome Hemachitra ${ }^{2^{*}}$ \\ Assistant Professor, Mangayarkarasi Arts and Science College for women, Madurai, TamilNadu, India ${ }^{1}$ \\ Lecturer, Sri Meenakshi Govt. Arts and Science College for women, Madurai, TamilNadu, India ${ }^{2}$
}

Received: 27-February-2020; Revised: 20-May-2020; Accepted: 22-May-2020

(C)2020 A.V. Seetha Lakshmi and H.Salome Hemachitra. This is an open access article distributed under the Creative Commons Attribution (CC BY) License, which permits unrestricted use, distribution, and reproduction in any medium, provided the original work is properly cited.

\begin{abstract}
Image processing has been functional to the numerous expansions of agricultural engineering in regulate to accomplish a quick accurate process. The procedure of physical categorization is leisurely and attains a level of bias, which is hard to be enumerated for typical type seed varieties. Seed examination and categorization can afford extra acquaintance in their creation, seeds superiority control and adulteration identification. Several techniques are utilized to resolve the struggle in perceiving and recognizing the standard type seed varieties, but the most objective is to categorize and recognize the Multifaceted Type Seed Varieties may be a quite difficult process, owing to its textural, shape and color patterns. These techniques do not provide an optimized and a correct depiction of the complex type seed varieties. The main objective of this work is to identify the complex type seed varieties for prospect fertilization within the field of agriculture. This context plans novel image processing systems to recognize, which incorporates an enhanced feature selection, and classification methodologies, which might optimize the exactness and reduce the time consumption of identifying the multifaceted type seed varieties. This novel technique provides efficient identification by feature selection and classification of those composite type seeds. The identification process, Adaptive Median Filter is employed for image enhancement; the edge detection for the image employs Sobel operator and Watershed Segmentation is used for the segmentation. Then Ant Colony Optimization (ACO) strategy is employed for the feature selection and Support Vector Machine (SVM) is employed in the classification process. The ACO based feature selection (ACOFS) provides ranges $8 \mathrm{~s}$ to 20 s of feature selection time for the dataset and the SVM classification provide $93.487 \%$ of accuracy while prediction.
\end{abstract}

\section{Keywords}

Complex type seed, Variety identification, Seed recognition, Image processing techniques.

\section{Introduction}

The utilization of great seeds is one of the most significant aspects in in any developing system of an agricultural field. This aspect has gotten more basic than any other time in recent forgiving enough food security to the rising number of individuals in the world, which is depended on to outperform nine billion continuously 2050. For the best harvest execution and good production yield, the selection of high yielding seed varieties without the essential characteristics like breaking obstruction, lodging, insect and diseases. The manufacture of superior seed is the foundation of any effective farming system.

\footnotetext{
*Author for correspondence
}

It is in like manner a decent showcasing tool for expanding the potential offer of yields, particularly in the present serious market. In general, the quality of the seeds is estimated from various perspectives, containing genomic and diseases free seed varieties, whatever other components that may influence seed execution in the field. Examples of the factors that affect seed quality are heat, mechanical damage, and pre-harvest growing in white wheat. Various characteristics like explicit substance synthesis or protection from specific diseases or creepy crawlies additionally add to the nature of seeds. Kilıç et al. [1] developed a quality assessment system based on a computer vision of beans dependent on color and size quality of tests. Skewness, average and variance values were resolved for each channel of RGB (red, green and blue) color position. Artificial Neural Networks (ANN) were utilized for color capability of 
the examples. The automated system had the option to classify white beans; yellow-green harmed beans, dark harmed beans, low harmed beans and highly harmed beans effectively [1]. Wang and Cheng describe that in the farming fields the authors require seeds just as weeds also. The extraction of the features of the image is performed using the PCA network. Enhanced PCA can offer classify and enhance the resultant weed seeds [2]. Tomasz, et al., the utilization of ANN and VIS-NIR (Visible-Near Infra-Red) reflection spectroscopy is to obtain the substance of fat and protein from the seeds [3].

It is said to be Image Processing plays main role in various applications and areas. Commonly master guidance may not be reasonable, lion's share times the accessibility of specialists and their administrations may devour time. Image Processing nearby the sincerity of correspondence systems can change the circumstance of getting the master advice well in time and at a reasonable expense since image, processing was a successful tool for investigation of parameters.

In the work, the identification and recognition of the complex type seed for the agricultural purpose are implemented with the employment of image processing methods. This process of employment would decrease the time consumption and optimize the exactness of the complex type of seed identification and recognition. The rest of this work is carried out with the subsequent structure.

The goals of this research are to present the automatic organization of agricultural seeds with the explosion of digital information through computing image vision processing. This work employs computer aided vision, image processing techniques for the identification and classification of canola seeds. Here analysis three types of seeds such as California burclover, Eleocharis, Carolinians.

The key objectives of this research work are initiated as follows:

- This proposed Complex Seed Identification System is a model system for the California burclover seed recognition system.

- The pulses are also known as California burclover, Eleocharis, Carolinians and have become a significant sow for Genetic and Genomic studies.

- In recent years, it is important to identify the productivity of seed. Due to the green revolution, there has been a decrease in the production of resulting in decreased seed production.

There are several identification systems are established for Cereal Grains and very little recognition systems for a pulse, but these systems are not strong for all pulse seed varieties and also could not recognize the seed variety accurately for complex type seeds. Thus, this proposed system emphases on the entire causes of the complex type seed variety system.

\section{Related review}

$\mathrm{Li}$, Jun 2018, all the strategies thought so far are utilized for the image classification just for accomplishing the seed classification. There exists a strong technique for classification of the image. This technique utilizes a deep stacking system (DSN) includes various SNNM with the Sigmoidal depiction work [4].

JayaBrindha, Classification is a regulated learning technique. Neural Networks need a gap of overfitting precarious because of which the speculation will be awful. Henceforth, SVM will be utilized to image classification dependent on the features mined from the images such as the edge, and so on [5].

Devaraj et al. [6] expressed that image processing systems were utilized to see plant corruptions. The leading target of this investigation was to build up a product system for automatic identification and order of plant corruptions. The image processing approach utilized different stages to perceive plant illnesses, for example, image acquisition, image preprocessing, segmentation, feature extraction, and classification.

Arya et al. [7] introduced an audit of different image processing strategies to see plant diseases. The significant goal of this investigation was to learn the contaminations. So as to recognize diseases, speed and exactness were distinguished as two basic perspectives.

Khmag et al. [8] planned to make a recognition framework for leaf dependent on leaf shape and centroid. An image processing calculation was created by using the variation to scaling shift, turn method, scaling approach, and sifting forms, just as SVM as the classifier for the leaf shape. The proposed strategy utilized 70 examples taken from the Flavia dataset and their geometrical and shape aspects were extricated and yielded an exactness of 
97.7\% which is the most elevated accomplishment announced in writing.

Automated species identification is moving toward the real world. In review the specialized the state of affairs on PC vision approaches for plant species identification, feature the fundamental research difficulties to defeat in giving appropriate devices, and end up with a conversation of open and future research pushes [9].

A technique for separating 15 aspects of leaf through a shrewd edge operator, and SVM as the classifier was accounted for by Salman et al. [10]. The removed aspects were curved region, filled region, edge $(\mathrm{P})$, unusualness (E), strength, and border proportion of measurement, direction, thin factor, degree, Euler number, and distance across, circularity, rectangularity, edge proportion of length and width, intricacy, and conservatives. The investigation utilized 2220 pictures from 22 plant species in the Flavia dataset and accomplished an exactness in 85 to $87 \%$ as it were.

In the paper [11] proposed System is sent to arrange Indian neighborhood leaves naturally utilizing profound learning. Profound Learning is a selflearning method utilized for enormous information and ongoing advancement in equipment and huge information has turned out as an ability. The paper has considered 10 diverse Indian plants leaves. The recognition rate of the proposed framework is $93 \%$.

\section{Proposed method}

This proposed technique focuses around the whole reasons of complex type seed variety identification and classification system. There are few systems that resolve the issue of identification and recognition of the typical type seed varieties, but the objective is to categorize and recognize the Complex Type of Seed Varieties is a quite tough process, because of its textural, shape and color patterns. These techniques do not provide an optimized and exact description of the complex type seed varieties.

Thus, the work starts a different system that incorporates a characterized technique to recognize the intricate variety and characterize the nature of the particular seed variety. This framework will physically pick some significant features to classify the varieties as opposed to testing whole features for a particular seed variety identification and classification. This will reduce the tedious and capacity assignment for the varieties of classified seeds. The following Figure 1 Overall architecture of the proposed model.

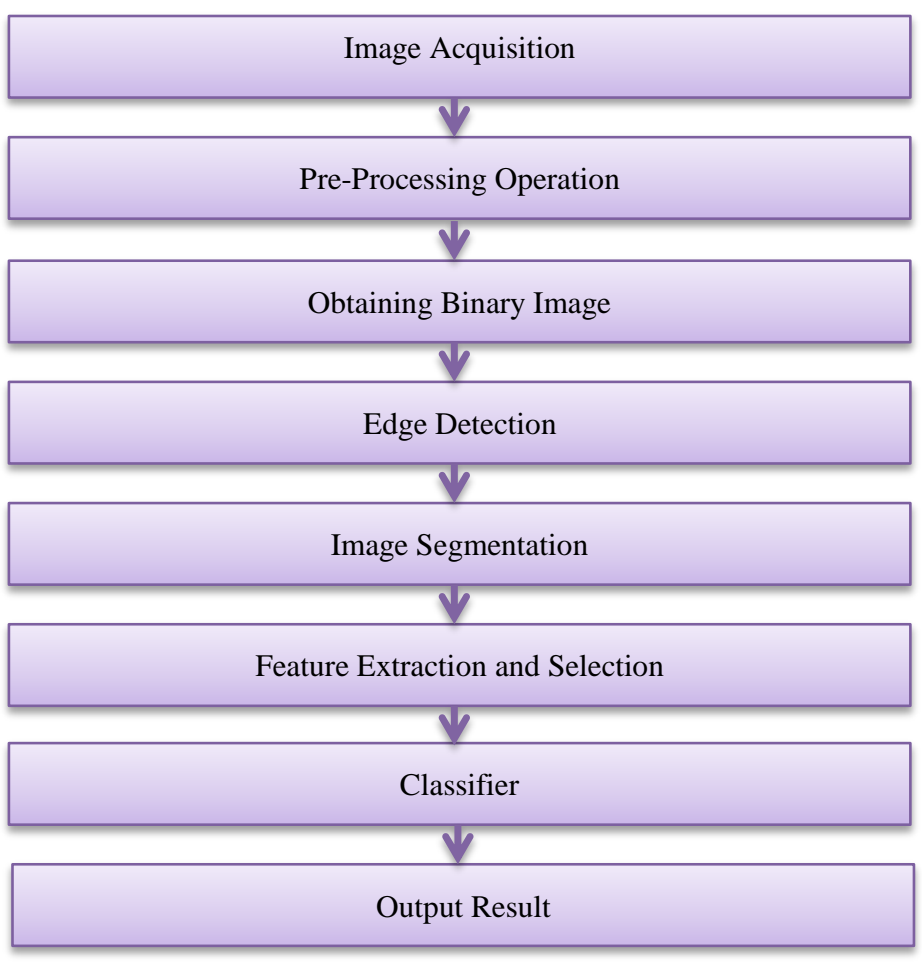

Figure 1 Overall architecture of the proposed model

The subdivision contains the procedure of preprocessing and region, discovery of the complex type of seed variety is labeled.

\section{i.Image acquisition}

An input image from color camera with a resolution of 640 × 480 pixels was utilized to acquire images. The setup of a chamber with a white table as a background for taking pictures. The following Figure 2 is Acquisition is accomplished with an appropriate camera. It uses utilizes various for different applications.

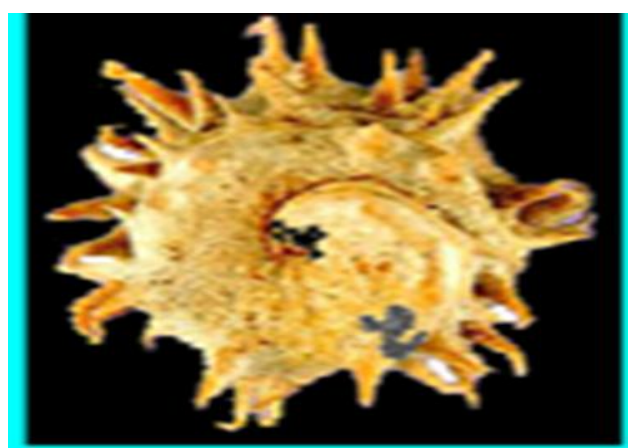

Figure 2 Sample complex type input seed image 


\section{ii.Pre-processing operation}

Any effort in the processing technique must be conscientiously adjusted to the particular natural product or seed type. Processing recommends a vulnerability of losing seeds, both by under and overtreatment. Under-treatment may fail, neglect to achieve the perfect result; over-treatment may offend the seeds with subsequent loss of reasonability or decreased stability. Both under and over-treatment may impact the hereditary synthesis of the seed lot.

In the field of image processing and computer vision, Noise Removal is one of the major critical issues. Images are usually degraded by impulsive noise because of noise sensors or channel transmission errors or faulty storage hardware. The goal of impulsive noise elimination is basically to smother the noise just as to safeguard the reliability of edges and detailed information. Noise filtering and image enhancement are the two-essential process in the digital image processing.

In this work, Adaptive Median Filter (AMF) uses varying window sizes for noise reduction. AMF fairs well at low and medium noise densities, however obscures the picture at high noise densities. The window size is increased which in turn obscures the picture. The Digital Image is enhanced in this section using Image Enhancement Techniques. In this section, using the Adaptive Median Filter strategy Enhancement process is performed. At first, the Image is converted to a grayscale for the visual perception of the image. Then the noises in the images are eliminated using the denoising filters. In this system, Mean Filter denoises the blur that happened in the image.

The following Figure 3 portrays the overall process flow of the identification phase, which incorporates the seed image edge detection and segmentation for the further recognition process.

\section{iii.Edge detection}

The edge identification methodology is its ability to segregate the specific edge focuses with extraordinary direction. Inscription about edge recognition has been uncovered in the previous three decades. Edge discovery methods change remarkable pictures into edge recognized pictures that advantage by the movements of dim tones in the picture. In image processing, particularly in computer vision, edge discovery treats the restriction of immense assortments of a dim level picture and the location of the physical and geometrical properties of objects of the scene.

The proposed algorithm mines the edge data of color images in RGB shading space with fixed edge threshold. The algorithm utilizes the Sobel edge detector for perceiving the edge. Another automatic edge threshold recognizable system dependent on histogram information is utilized for assessing the edge threshold. The system is applied to an enormous number of images and the result shows that the algorithm produces absorbing outcomes when contrasted with a portion of the current edge recognition methods.

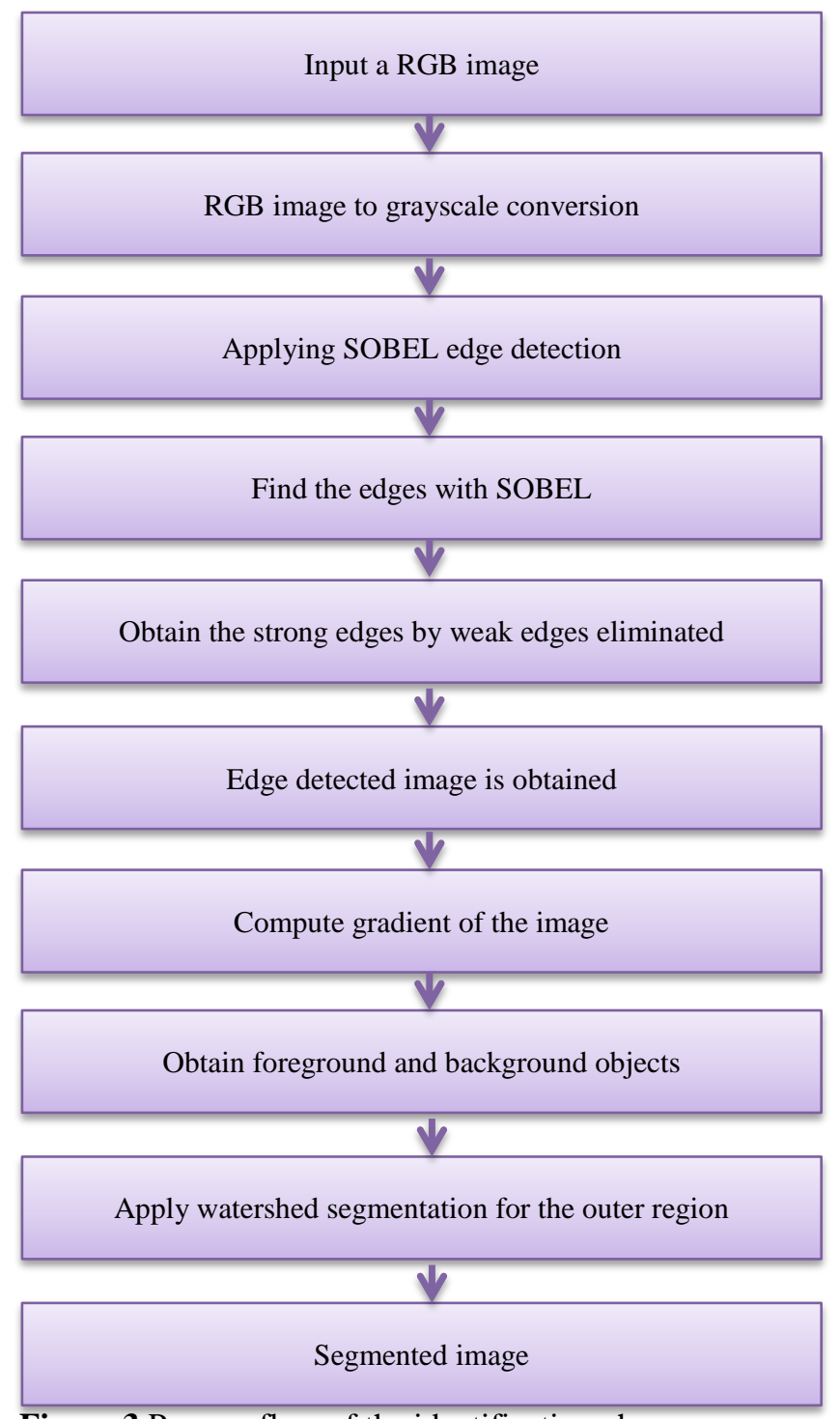

Figure 3 Process flow of the identification phase 


\section{Algorithm SOBEL}

Input: The following A Sample images.

Output: Detected Edges.

1: Distinguish the input image.

2: Turn the color image into a grayscale image

3: Employ the mask Gx, Gy to the input image.

4: Apply the Sobel edge detection method and the gradient values

5: Operate the masks Gx, Gy independently on the input image.

6: Results are joined to trace the absolute magnitude of the gradient.

7: The absolute magnitude is the detected edge.

The following Figure 4 Edge detected image.

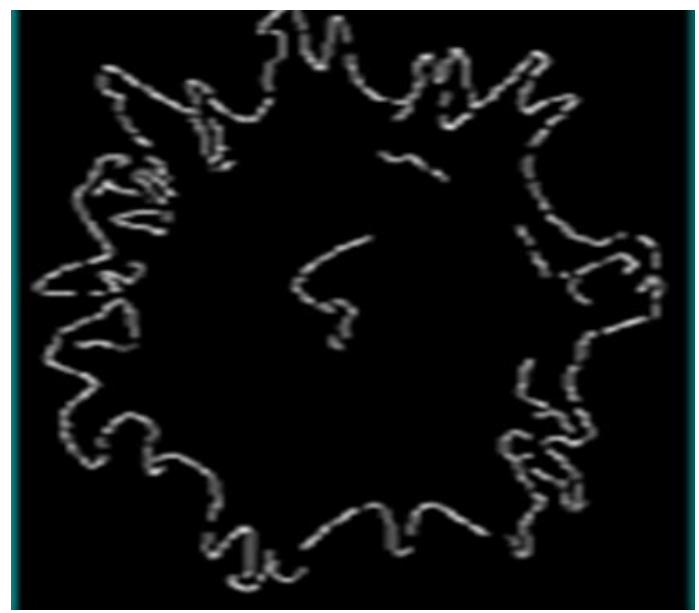

Figure 4 Edge detected image

\section{iv.Image segmentation}

In this section, the segmentation process is performed by utilizing the technique of Watershed Segmentation to fill the inner region of the image. In Watershed Segmentation, compute the foreground view object, which connects masses of pixels inside each of the foreground objects that utilizes the Morphological opening and closing by reconstruction to tidy up the picture. Evaluate the background objects. In the cleaned-up image, the background pixels are in darkness. Computer the watershed renovates of the segmentation work. The function 'imposemin' can be used to alter an image so that it has regional minima only in certain desired locations. This strategy chooses the color that is assigned to each object, based on the number of objects in the label matrix and a range of colors in the color map.

The following Figure 5 Finally, the proposed strategy gets a fundamentally similar analysis of the complex type of seed images in all types of seed images when compared to the analysis of the seed identification.

\section{Algorithm watershed}

1: Read an image and convert into a matrix with representation of 0 's and 1's. Those intensity values of the pixels are changed to 0 when intensity is higher or equivalent to 195 and others are changed to 1.

2: All connected components that have fewer than 20 pixels are removed from the Binary Image.

3: Perform the flood-fill operation by filling the 'holes' in the background image.

4: The matrix is changing inversely by changing 0 's to 1's and vice-versa.

5: The Euclidean distance is computed for the binary image.

6: Now calculate the label matrix recognizing the watershed regions.

7: The components named ' 1 ' belong to the first watershed region; the components named ' 2 ' belong to the second watershed region and so on.

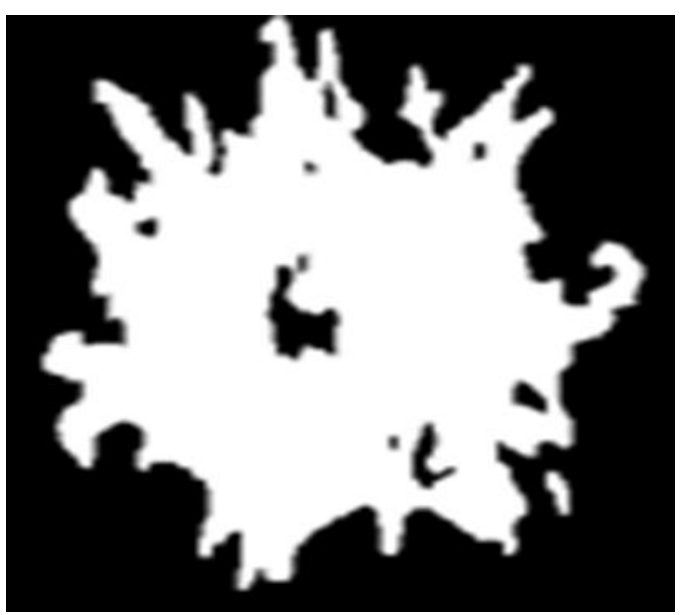

Figure 5 Inner region detected image

\section{v.Feature extraction and selection}

Feature extraction is an imperative phase in image classification. It permits representing the content of images as effortlessly as possible. Before receiving features, various image-preprocessing techniques are applied to the image. After that, feature extraction techniques are applied to extract features that will be beneficial in classifying and recognition of images. Feature extraction methods are helpful in frequent image-processing applications. Image dependent features are texture features, color features and shape features are as follows: 
Color Features: The color features of an image represent through various color models to present the color information.

Texture Features: The one of the essential properties of the image is texture. This helps in a retrieval process of the region of the image. It can be used to classify textured images from non-textured images.

Shape Features: For image content description, the shape is a significant visual feature and one of the primitive features.

Higher-dimensional data commonly will diminish Accuracy of the Classification. As indicated by the Hughes phenomenon, the specified training sample size for classification grows exponentially as the variety of spectral bands increase. Dimensionality reduction additionally improves classification time. To decrease training data size, a reduction of the number of dimensions is required. Dimensionality Reduction is furthermore expected to kill extremely related to bands. Here Dimensionality Reduction will be sorted as Feature selection.

\section{ACO based Feature Selection}

Feature extraction and selection decrease are essential stages all through pattern classification. During this investigation, disregarding the feature, set was not redundant and giving adequate results, utilizing the main features to increase the recognition rate. Eliminating some of the features allow the classifier to attain the more generalization performance.

ACO is an iterative, Probabilistic Meta-Heuristic for discovering answers to combinatorial enhancement issues. While foraging, the ants convey in a roundabout way using their pheromone that they use to stamp their different ways and which pull in different ants. In the ACO technique, artificial ants (agents) create virtual emission to refresh their way through the decision chart. The agents utilize the pheromone markings of past reasonable agents as a technique for orientation when making their own chooses to search for the briefest method for each possible other choice. The following Figure 6 Antcolony optimization flow diagram.

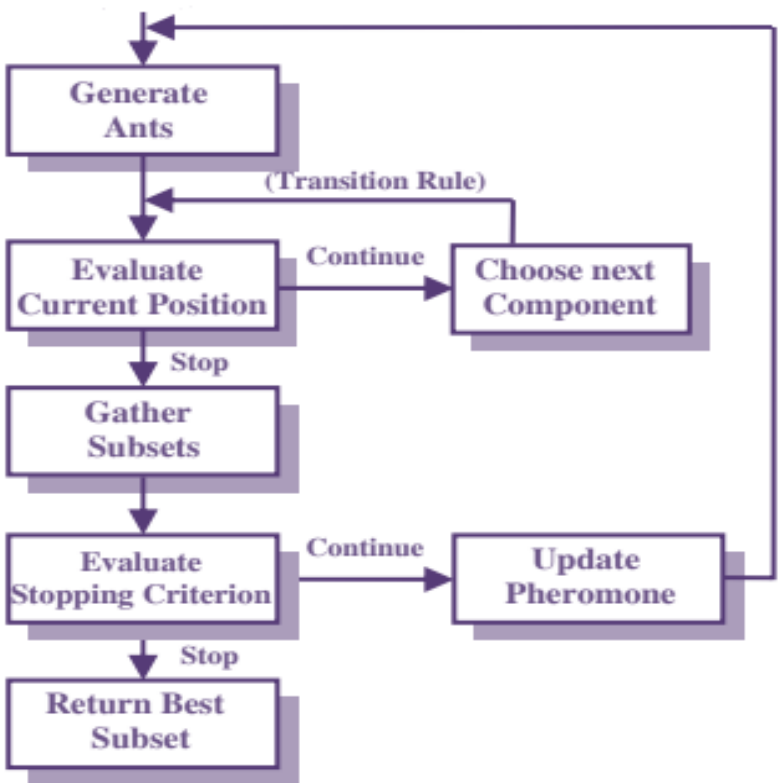

Figure 6 Ant-colony optimization flow diagram

The procedure starts by producing variety ' $\mathrm{k}$ ' ants, which are then provided the graph with random ants. As another option, various ants to put on the graph is also set equivalent of the extracted features inside the data; every ant begins path construction at a distinctive feature. The traversal of the edge probability is repeated until the condition is satisfied. Then the resultant subsets are collected for the further assessment.

\section{Algorithm ACOFS}

Input: D-Dataset

AI-Input for ACO

$\alpha, \beta, p, r$-number of ants ACO's parameters

1: Set the same value for pheromone and heuristic information

2: for each i from number_of_epochs

3: \{

4: for each $\mathrm{j}$ from number_of_ants

5: ant $_{\mathrm{j}}$ connects consecutively feature path indices

6: $\}$

7: The associated training set is constructed for every path

8: The fitness value is computed for every path using SVM classifier

9: The path consistent to the best fitness is placed

10: The pheromone stages are reorganized

11: If the ' $n$ ' number of epochs is reached then the loop terminates and stops 


\section{vi. Classifier}

Seed classification is a procedure wherein various assortments of seeds are categorized into dissimilar classes based on their morphological features. Computers can use machine vision technologies in combination with a camera and artificial intelligence software to achieve image recognition. Classification in SVM is an example model of supervised learning to analyze whether the system is performing in the correct way or not is demonstrated with the help of best-known labels. This data points to the desired response, validating the accuracy of the system, or be used to figure out how to act appropriately.

The classifier is employed to classify complex type seed images from typical type seeds. SVM takes many images and predicts for each info picture has a place with which of the classes of classifications. The aim of SVM is to create hyperplane that isolates classifications with the most holes between them. In this proposed system, output of feature selected is given as input to SVM classifier that takes training data, testing data and grouping information that classifies whether given information of the input picture. The training information, samples along the hyperplanes near the category limit are known as support vectors. The margin is the distance between the support vectors and the class boundary hyperplanes. The SVM has upheld the build of decision planes that define decision boundaries. SVM could useful strategy for data classification. A classification task normally involves training and testing information that comprises of some data instances. Every instance within the training set contains one class labels and several other features.

\section{v. Performance and comparative analysis}

In this section, the performance and comparative analysis of the procedures employed in the identification and recognition of the multi-faceted seeds. The performance metrics like PSNR, SNR, and edge detection metrics are assessed.

\section{a) PSNR Value}

The evaluation of the PSNR value is shown in Figure 7. The figure portrays that the Wiener filter affords a high PSNR rate than other filters.

The SNR evaluation for the several noises is depicted in Figure 8. The Figure illustrates that the wiener filter provides high SNR value for several noises than other approaches.

Figure 9 illustrates the performance metrics Entropy and Correlation Coefficient evaluation for edge detection procedures. The figure portrays that the canny method offers better results than other approaches.

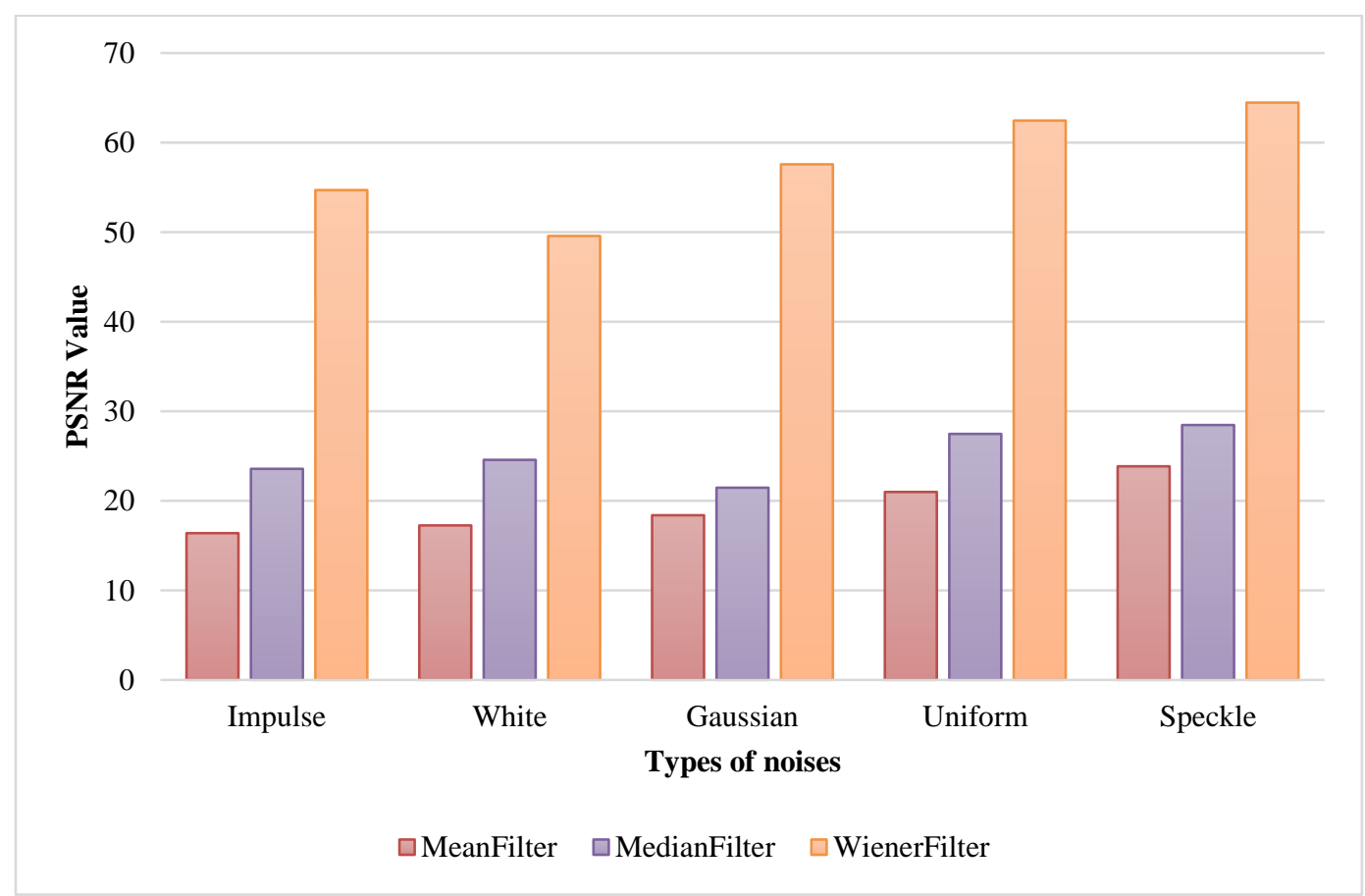

Figure 7 PSNR value evaluation 
A.V. Seetha Lakshmi and H.Salome Hemachitra

b) The SNR value

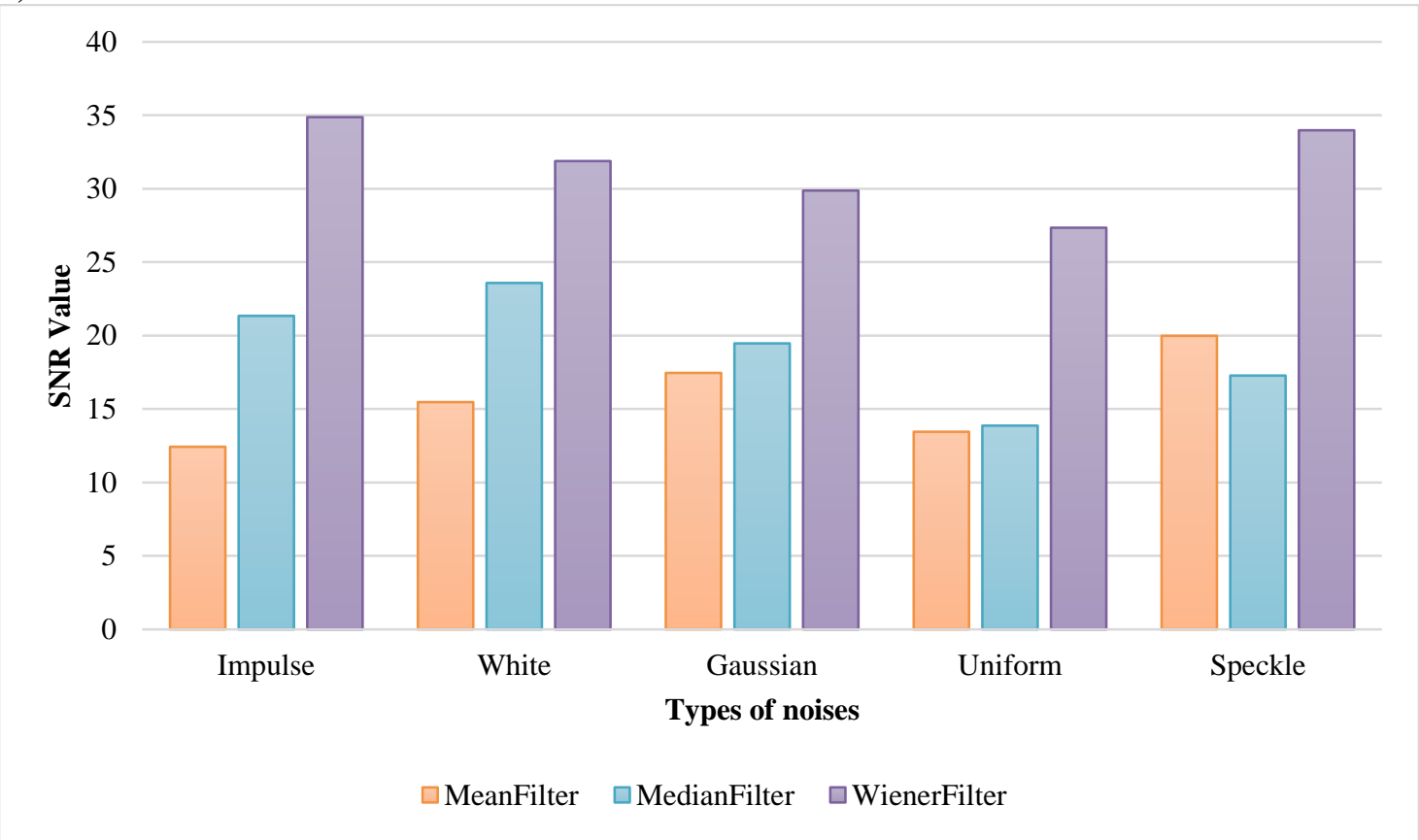

Figure 8 SNR value evaluation

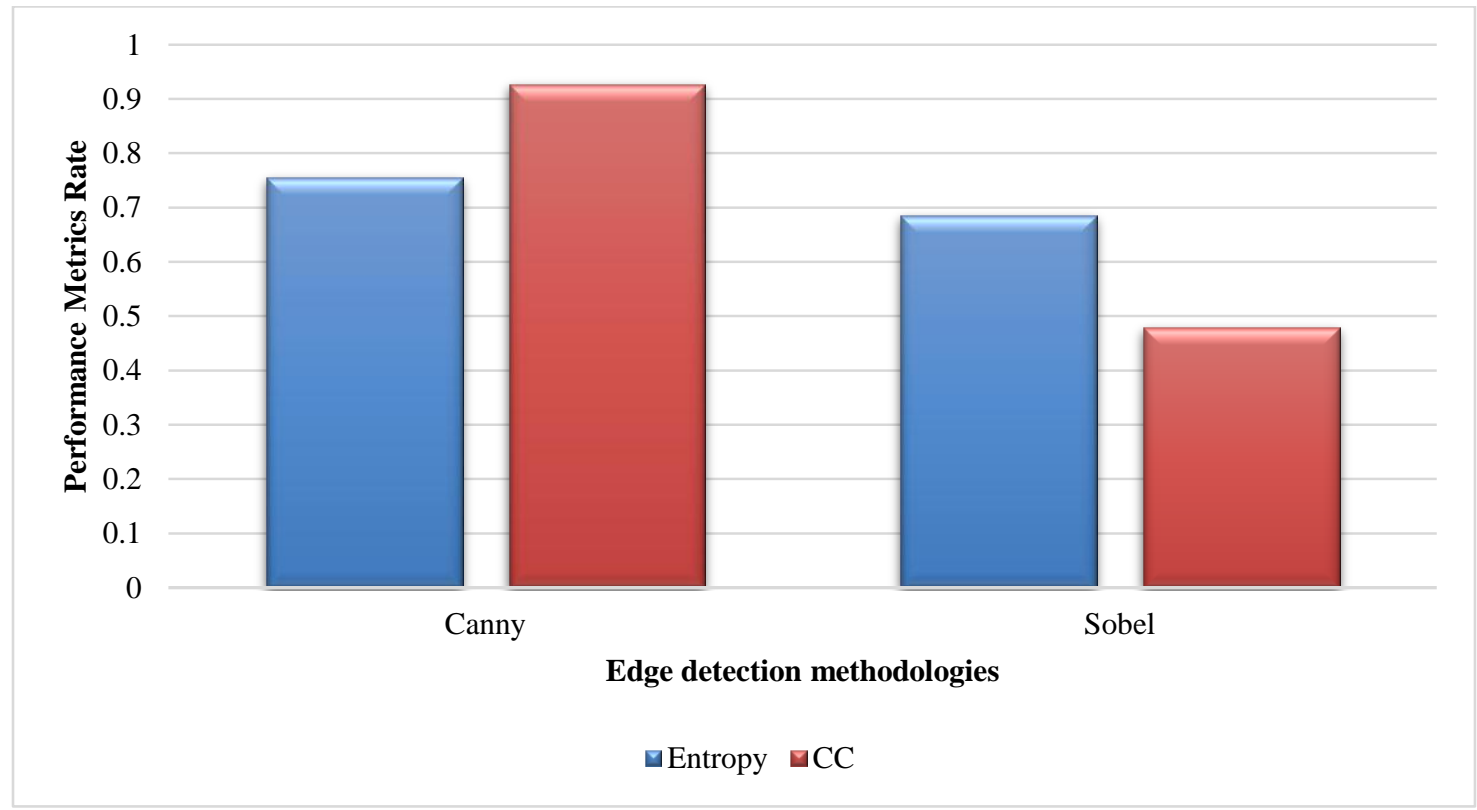

Figure 9 Performance metrics evaluation for edge detection methodologies 


\section{Conclusion}

The main objective of the work is Complex Type Seed Identification using feature extraction and selection techniques with image processing. The seed image is input to the system for the enhancement and filtering the noises for the further identification process. Then the enhanced image is processed to obtain the image edges and segmentation. The segmented image is utilized for the identification of the seed variety. The identified image is characterized by the extraction of the features and significant features by using feature selection strategy to increase the classification rate. This framework could be adjusted for limiting the human visual appraisal of Complex Type seed engaged with seed recognizable and confirmation of the normal result of the estate. These work points are cultivated by two principle goals: the Complex Type Seed Identification System and the Recognition System. In future the proposed system can recognize which suitable soil for the seed and can recommend suitable fertilizers and weather conditions for the seeds. The proposed system can be extended with the quality identification and disease, identification of the seed.

\section{Acknowledgment \\ None.}

\section{Conflicts of interest}

The authors have no conflicts of interest to declare.

\section{References}

[1] Kılıç K, Boyacı IH, Köksel H, Küsmenoğlu İ. A classification system for beans using computer vision system and artificial neural networks. Journal of Food Engineering. 2007; 78(3):897-904.

[2] Xinshao W, Cheng C. Weed seeds classification based on PCANet deep learning baseline. In Asia-pacific signal and information processing association annual summit and conference (APSIPA) 2015 (pp. 408-15). IEEE.

[3] Wojciechowski T, Niedbała G, Czechlowski M, Nawrocka JR, Piechnik L, Niemann J. Rapeseed seeds quality classification with usage of VIS-NIR fiber optic probe and artificial neural networks. In international conference on optoelectronics and image processing (ICOIP) 2016 (pp. 44-8). IEEE.

[4] Li J, Chang H, Yang J, Luo W, Fu Y. Visual representation and classification by learning group sparse deep stacking network. IEEE Transactions on Image Processing. 2017; 27(1):464-76.

[5] JayaBrindha G, Subbu EG. Ant colony technique for optimizing the order of cascaded SVM classifier for sunflower seed classification. Transactions on Emerging Topics in Computational Intelligence. 2017; 2(1):78-88.
[6] Devaraj A, Rathan K, Jaahnavi S, Indira K. Identification of plant disease using image processing technique. In international conference on communication and signal processing (ICCSP) 2019 (pp. 749-53). IEEE.

[7] Arya MS, Anjali K, Unni D. Detection of unhealthy plant leaves using image processing and genetic algorithm with Arduino. In international conference on power, signals, control and computation (EPSCICON) 2018 (pp. 1-5). IEEE.

[8] Khmag A, Al-Haddad SR, Kamarudin N. Recognition system for leaf images based on its leaf contour and centroid. In student conference on research and development (SCOReD) 2017 (pp. 467-72). IEEE.

[9] Wäldchen J, Rzanny M, Seeland M, Mäder P. Automated plant species identification-trends and future directions. PLoS Computational Biology. 2018.

[10] Salman A, Semwal A, Bhatt U, Thakkar VM. Leaf classification and identification using canny edge detector and SVM classifier. In international conference on inventive systems and control (ICISC) 2017 (pp. 1-4). IEEE.

[11] Naglot D, Kasliwal PS, Gaikwad SJ, Agrawal ND. Indian plant recognition system using convolutional neural network. International Journal of Computer Sciences and Engineering. 2019; 7(6):276-80.

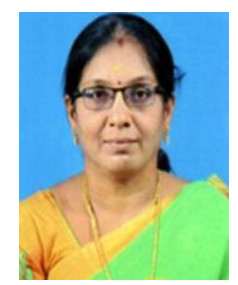

Dr. A.V. Seethalakshmi, Assistant Professor in Computer Science, Mangayarkarasi College of Arts \& Science for women, Paravai, Madurai. She received here MCA Degree from Madurai kamraj University, Madurai. She has been awarded her Ph.D Degree in Computer Applications in the year 2018. She has $18+$ years of experience in teaching field. She has published $10+$ research papers in journals and conferences. She has interests in domains like Data Mining, Image Processing and Networks.

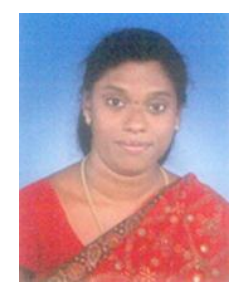

Dr. H.Salome Hemachitra, Lecturer in Computer Science, Sri Meenakshi Government Arts College for Women, Madurai. She completed her UG Degree (B.Sc (Physics)) LDC, Madurai in 1999, and PG Degree (M.Sc (Computer Science)) in MKU College, Madurai in 2001. She has been awarded her Ph.D Degree in Computer Science before 2 years and $15+$ years of experience in teaching field. She has published $13+$ research papers in journals and conferences. She has interests in domains like, Image Processing, Data Mining and Networks.

Email: salomechitra_2@yahoo.com 